\title{
Comportamiento Sexual y factores asociados en Estudiantes de Odontología
}

\author{
Antonio Díaz Caballero ${ }^{a}$, Katherine Arrieta Vergara ${ }^{b}$, Farith González Martínez $^{c}$
}

\begin{abstract}
a Odontólogo, Magíster en Educación, Profesor Titular en el Departamento de Medicina Oral, Facultad de Odontología,

Universidad de Cartagena, Colombia.

b Odontóloga, Magíster en Salud Pública,

Asistente en

el Departamento de Odontología Preventiva

y Social, Facultad de Odontología, Universidad de Cartagena, Colombia.
\end{abstract}

c Odontólogo, Magíster en Salud Pública, Profesor Asistente en el Departamento de Investigación, Facultad de Odontología, Universidad de Cartagena, Colombia.

Correspondencia:

Antonio Díaz Caballero,

Facultad de Odontología, Universidad de Cartagena, Campus de la Salud, Departamento de investigación,

Barrio Zaragocilla,

Cartagena de Indias,

Colombia.

Correo electrónico: antoniodiazc@yahoo.com. Telf.: 05756698172, Ext. 110.

Recibido el 15 de diciembre de 2009.

Aceptado para su publicación el 12 de marzo de 2010.

\section{RESUMEN}

Objetivo. Describir el comportamiento sexual y evaluar los factores asociados en estudiantes de Odontología de la ciudad de Cartagena (Colombia).

Métodos. Se realizó un estudio de corte transversal en una muestra de 381 estudiantes de Odontología de la ciudad de Cartagena, seleccionados en forma aleatoria proporcional al tamaño de cada semestre cursado. Se aplicó un cuestionario para medir las siguientes variables: haber tenido relaciones sexuales, edad, género, estrato, semestre, consumo de alcohol y drogas. Los datos fueron analizados a partir de proporciones, realizándose un análisis bivariado a través de razones de disparidad y un análisis multivariable por medio de regresión logística.

Resultados. La prevalencia de haber tenido relaciones sexuales fue del $64,6 \%$, siendo mayor en varones $(81,8 \%)$ que en mujeres $(53,6 \%)$. La edad promedio de la primera relación sexual fue de 16,9 años (DE: 2,6). En el análisis multivariable por género, haber tenido relaciones sexuales en los varones se asoció con el consumo de alcohol (OR: 1,85; $p=$ $0,000)$, mientras que en las mujeres, se asoció con estar cursando semestres superiores (OR: 2,70; $p=0,003$ ), edad mayor de 18 años (OR: 2,$45 ; p=0,01$ ) y el consumo de alcohol (OR: 1,$83 ; p=0,03$ ).

Conclusiones. Los comportamientos sexuales en estudiantes de Odontología de la ciudad de Cartagena difieren entre varones y mujeres, encontrándose variaciones también entre los factores asociados, siendo más influyentes en las mujeres con respecto a su interacción para explicar el fenómeno.

Palabras clave. Conducta Sexual, Estudiantes de Odontología, Género.

\section{ABSTRACT}

\section{Sexual behavior and associated factors in dental students}

Objective. To describe sexual behavioral and associated factors in assessing dental students from the city of Cartagena (Colombia),

Methods. It was conducted a cross-sectional study with a sample of 381 students of dentistry, randomly selected proportional to the size of each semester completed. A questionnaire was administered to measure the variables having sex, age, gender, stratum, semester, alcohol and drugs. Data were analyzed from proportions, carrying out bivariate analysis through the grounds of disparity and a multivariate analysis using logistic regression.

Results. The prevalence of having had sex was $64.5 \%$, being higher in males $(81.8 \%)$ than females $(53.6 \%)$. The average age at first intercourse was 16.9 years $(S D=2.6)$. In multivariate analysis by gender, having sex in men was associated with alcohol consumption $(O R=1.85 ; P=0.000)$, whereas in women, is associated with being enrolled in higher semesters $(O R=2.70 ; P=0.003)$, age over 18 years $(O R=2.45 ; P=0.01)$ and alcohol $(\mathrm{OR}=1.83 ; \mathrm{P}=0.03)$.

Conclusions. Sexual behaviors among students of dentistry in the city of Cartagena differ between men and women, also found variations between the related factors being more influential in women with regard to their interaction to explain the phenomenon.

Key words. Sexual Behavioral, Dental Students, Gender Identity. 


\section{INTRODUCCIÓN}

El comportamiento sexual de un individuo es entendido como una expresión conductual de la sexualidad personal, donde el componente erótico es el más evidente y en la mayoría de los casos está ligada a la obtención de placer ${ }^{1}$. La falta de comunicación por parte de padres y maestros y los conocimientos obtenidos dentro del círculo social en que están inmersos los adolescentes lleva a los jóvenes a iniciar más tempranamente las relaciones sexuales sin la debida protección, trayendo como consecuencia los embarazos no planificados y las enfermedades de transmisión sexual ${ }^{2,3}$. Éstas parecen ser las razones más convincentes para promover el uso de los preservativos entre los adolescentes e implementar programas educativos que respondan a fortalecer unas prácticas sexuales con mayor responsabilidad 4 .

A nivel de Colombia, Profamilia, el Instituto del Seguro Social y el Instituto Nacional de Cancerología realizaron en $1993^{5}$ la encuesta de conocimientos, actitudes y prácticas para explorar los comportamientos de riesgo en la salud de los adolescentes entre 12 y 17 años, encontrando que el $17 \%$ de ellos manifestó haber tenido relaciones sexuales, siendo en varones más frecuente que en mujeres. La edad promedio para la primera relación sexual fue de 13,4 años en varones y 14,8 para las mujeres. Por otro lado, los resultados de la encuesta nacional de Demografía y Salud en Colombia 2005 señalaron que el $11 \%$ de las mujeres consultadas tuvieron su primera relación sexual antes de cumplir 15 años y el $46 \%$ antes de cumplir los 18 años ${ }^{6}$.

Hoy es claro que a nivel regional el problema crece cada día más y no es ajeno a las instituciones universitarias, donde se hacen continuas investigaciones para conocer el comportamiento sexual y las actitudes que los estudiantes toman frente a la búsqueda de soluciones. En este sentido, Ceballos ${ }^{7}$ et al en 2006 en la Universidad de Magdalena encontraron que el $45,6 \%$ de los estudiantes tienen relaciones sexuales y de éstos el 95,6 \% conoce métodos anticonceptivos, siendo la edad promedio de inicio de la actividad sexual de 16 años.

Si bien se tienen datos de algunas poblaciones de la región Caribe colombiana, en la ciudad de Cartagena no hay reportes del comportamiento sexual en estudiantes universitarios, menos aún en estudiantes de Odontología, tópico este muy importante de estudiar debido al creciente número de embarazos que se ha observado en el último año en esta población, según datos obtenidos de la oficina de Bienestar Universitario de la Universidad de Cartagena. Desde esta perspectiva, el objetivo de este estudio fue describir el comportamiento sexual y evaluar los factores asociados en estudiantes de Odontología de la ciudad de Cartagena (Colombia).

\section{MATERIAL Y MÉTODO}

Diseño de corte transversal con un muestreo aleatorio estratificado, realizando una selección proporcional al tamaño de cada semestre cursado. La población fueron los estudiantes matriculados en la facultad de Odontología de la universidad de Cartagena para el segundo semestre del año 2007.

El calculo del tamaño de la muestra se calculó teniendo en cuenta una confianza del $95 \%$, un error tipo I del $5 \%$ y un error tipo II del $20 \%$. Además, la frecuencia esperada del evento en estudio (haber tenido relaciones sexuales) en la población no expuesta a los factores asociados fue del $25 \%$. Asumiendo un OR estimado de 1,3 , se obtuvo como resultado 381 estudiantes.

Previamente a la selección de la muestra, se tuvo en cuenta que los estudiantes aceptaran participar en el estudio diligenciando un consentimiento informado por escrito, en donde se explicaba el objeto de esta información y que los investigadores garantizarían la confidencialidad de la información. Todo esto según la normatividad ética vigente en nuestro país en caso de investigaciones en seres humanos $^{8}$. Por otro lado, el proyecto fue catalogado sin riesgo alguno y aprobado por el Comité Institucional de Ética de la Universidad de Cartagena.

Para medir el comportamiento sexual en los participantes se diseñó un cuestionario con 13 preguntas, de las cuales ocho eran estructuradas politómicas, dos dicotómicas y las tres restantes no estructuradas. Con este instrumento se quiso conocer el comportamiento sexual en estudiantes de Odontología, indagando en tópicos como haber tenido relaciones sexuales, edad de la primera relación, relaciones planeadas, orientación sexual, número de parejas sexuales, uso y tipo de anticonceptivo y consumo de sustancias psicoactivas. Este formato fue examinado inicialmente por dos expertos con el fin de evidenciar su validez de apariencia. Posteriormente fue aplicado en una prueba piloto para valorar las características de cada una de las preguntas y garantizar la comprensión por parte de los participantes. Además, las respuestas obtenidas fueron cotejadas con la teoría. El cuestionario fue autodiligenciado por los participantes en el aula de clases haciéndolo inicialmente desde los primeros semestres hasta culminar con el último grado.

Para la finalidad del análisis la variable de salida fue el haber tenido relaciones sexuales y las variables explicatorias fueron: género, edad, estrato, semestre, orientación sexual, consumo de alcohol, consumo de drogas. Además, se realizó un segundo análisis con la variable haber tenido relaciones sexuales no planeadas. Para el análisis bivariado y multivariado todas las variables fueron dicotomizadas. 
Los estimadores fueron calculados teniendo en cuenta el diseño muestral a través del paquete estadístico STATA versión para Windows 10.0, y se tuvieron estimadores crudos y ajustados por regre$\operatorname{sión}^{9}$. Con la variable género se pudo estratificar la condición haber tenido relaciones sexuales. Para determinar las asociaciones se utilizaron las razones de disparidad (OR), con intervalos de confianza del $95 \%$. Para controlar las posibles variables confusoras se realizó un análisis multivariable a través de regresión logística, siguiendo las recomendaciones de Greenland ${ }^{10}$. En todo el análisis el límite para obtener resultados estadísticamente significativos fueron los valores de probabilidad menores del $5 \%$.

\section{RESULTADOS}

Un total de 381 participantes diligenciaron el cuestionario de forma satisfactoria. El promedio de edad fue de 20,4 años (DE: 2,6), observándose un valor mínimo de 16 y máximo de 30 años. Los rangos entre 19 y $21(42,5 \%)$ y entre 16 y $18(26,5 \%)$ fueron los más frecuentes. En cuanto al género, el 38,8 \% fueron varones y el $61,2 \%$ mujeres. Con respecto a la variable semestre, de los diez cursos evaluados, el $40,5 \%$ de los estudiantes estaban cursando entre primero y cuarto semestre y el 59,5\% entre quinto y décimo, encontrándose el mayor número de estudiantes en este último curso (12,3\%). Con respecto al estado civil, el $96,6 \%$ se encontraban solteros. En cuanto al estrato socioeconómico, de los seis niveles incluidos, los que más participantes aportaron fueron el tres con $42,3 \%$ y el dos con el $32,6 \%$. Por último, el $61,4 \%$ de los encuestados consumía alcohol y el 1,8\% había consumido alguna sustancia ilegal (marihuana, cocaína y otras).

La prevalencia de haber tenido relaciones sexuales fue del $64,6 \%$, siendo mayor en varones $(81,1 \%)$ que en mujeres $(53,6 \%), p=0,000$. La edad promedio de la primera relación fue a los 16,9 años (DE: 2,6). Al analizar por género, la edad en varones fue del 15,9 (DE: 2,5) y la de mujeres de 17,9 (DE: 2,3$)(p=0,0004)$. De este grupo de encuestados, el 62,2 \% manifestó que sus relaciones no

\begin{tabular}{|c|c|c|c|c|}
\hline & Varones (\%) & Mujeres (\%) & Total $(\%)^{*}$ & Valor $\mathbf{P}$ \\
\hline \multicolumn{5}{|l|}{ Relaciones sexuales } \\
\hline $\mathrm{Si}$ & $120(81,1)$ & $126(53,6)$ & $246(64,6)$ & \\
\hline No & $28(18,9)$ & $107(46,4)$ & $135(35,4)$ & 0,000 \\
\hline \multicolumn{5}{|c|}{ Relaciones no planeadas } \\
\hline $\mathrm{Si}$ & $85(70,8)$ & $68(54,0)$ & $153(62,2)$ & \\
\hline No & $35(29,2)$ & $58(46,0)$ & $93(37,8)$ & 0,006 \\
\hline \multicolumn{5}{|l|}{ Parejas sexuales } \\
\hline Mayor de tres & $68(56,7)$ & $18(14,3)$ & $86(34,9)$ & \\
\hline Tres o menos & $52(43,3)$ & $108(85,7)$ & $60(65,1)$ & 0,000 \\
\hline \multicolumn{5}{|l|}{ Orientación sexual } \\
\hline Heterosexual & $115(77,7)$ & $19(51,1)$ & $234(61,4)$ & \\
\hline Bisexual & $2(1,4)$ & $3(1,3)$ & $5(1,3)$ & \\
\hline Homosexual & $3(2,0)$ & $4(1,7)$ & $7(1,8)$ & 0,000 \\
\hline NA & $28(18,9)$ & $107(45,9)$ & $135(35,4)$ & \\
\hline \multicolumn{5}{|c|}{ Uso de anticonceptivos } \\
\hline $\mathrm{Si}$ & $55(45,8)$ & $73(57,9)$ & $118(48,0)$ & \\
\hline No & $65(54,2)$ & $53(42,1)$ & $128(52,0)$ & 0,004 \\
\hline \multicolumn{5}{|l|}{$\begin{array}{l}\text { Tipo de anticonceptivo } \\
\text { usado habitualmente }\end{array}$} \\
\hline Condón & $70(47,3)$ & $46(19,7)$ & $116(30,0)$ & \\
\hline Inyectable & $3(2,0)$ & $14(6,0)$ & $17(4,0)$ & \\
\hline Ritmo & $4(2,7)$ & $13(5,6)$ & $17(4,0)$ & \\
\hline Coito interrumpido & $4(2,7)$ & $7(3,0)$ & $11(3,0)$ & \\
\hline Píldora anticonceptiva & $29(19,6)$ & $22(9,4)$ & $51(8,0)$ & \\
\hline Otros & $8(5,4)$ & $21(9,0)$ & $29(1,0)$ & \\
\hline Ninguno & $2(1,4)$ & $3(1,3)$ & $5(13,0)$ & 0,000 \\
\hline $\mathrm{NA}$ & $28(18,9)$ & $107(45,9)$ & $135(35,0)$ & \\
\hline
\end{tabular}

Tabla 1. Comportamiento sexual de los estudiantes de Odontología según el Género. *Estimadores ajustados por muestreo 


\begin{tabular}{lccccccc}
\hline & \multicolumn{3}{c}{ Relaciones sexuales } & \multicolumn{3}{c}{ Bivariado } & \multicolumn{3}{c}{ Multivariado } \\
\hline & SI & NO & Total & OR & IC 95\% & OR & IC 95\% \\
Edad mayor de 18 & 204 & 76 & 280 & $4,09^{\mathrm{a}}$ & $2,53-6,60$ & $2,18^{\mathrm{b}}$ & $1,19-3,97$ \\
Género Masculino & 119 & 29 & 148 & $2,54^{\mathrm{a}}$ & $2,19-5,73$ & $3,29^{\mathrm{a}}$ & $1,96-5,51$ \\
Estrato Medio/Alto & 152 & 74 & 226 & 1,40 & $0,92-2,14$ & & \\
Semestre VI a X & 142 & 44 & 186 & $2,94^{\mathrm{a}}$ & $1,89-4,56$ & $2,10^{\mathrm{c}}$ & $1,20-3,66$ \\
Consumo de Alcohol & 174 & 60 & 234 & $3,19 \mathrm{a}$ & $2,06-4,93$ & $2,57^{\mathrm{a}}$ & $1,60-4,13$ \\
Consumo de Drogas & 7 & 0 & 7 & & & &
\end{tabular}

Tabla 2. Factores asociados con haber tenido relaciones sexuales en estudiantes de Odontología. ${ }^{a} P=0,000,{ }^{b} P=0,01,{ }^{c} P=0,009$.

fueron planeadas, observando en varones la mas alta prevalencia con el 70,8\% $(p=0,006)$. Además, el $34,9 \%$ de los encuestados que reportaron haber tenido relaciones sexuales manifestó haber tenido más de tres parejas sexuales, siendo el condón el anticonceptivo de mayor uso con un $30,0 \%$ (tabla1).

Con respecto al análisis bivariado, se encontró asociación con la mayor fuerza estadística entre la variable haber tenido relaciones sexuales y la edad mayor de 18 años (OR: 4,9; IC 95\%: 2,53-6,60; $p=$ 0,000 ), el consumo de alcohol (OR: 3,19 ; IC $95 \%$ : $2,06-4,93 ; p=0,000)$ y los semestres superiores (OR: 2,94; IC 95\%: 1,89-4,56; $p=0,000$ ) (tabla 2). Con respecto a la variable haber tenido relaciones sexuales no planeadas, la más alta fuerza estadística se observó con el uso de anticonceptivos (OR:
3,82; IC 95\%: 2,19-6,68; $p=0,000$ ) (tabla 3).

En cuanto al análisis multivariable, el mejor modelo final mostró asociaciones que explican haber tenido relaciones sexuales a través de cuatro indicadores; género varones (OR: 3,$29 ; p=0,000$ ), consumo de alcohol (OR: 2,57; $p=0,000$ ), edad mayor de 18 años (OR: 2,$18 ; p=0,01)$ y semestres superiores (OR: 2,10; $p=0,009$ ) (tabla 2). Al realizar el análisis multivariable independientemente por género, en varones solo el consumo de alcohol fue estadísticamente significativo (OR: 1,$85 ; p=0,000)$, mientras que en las mujeres, tres variables explicaron haber tenido relaciones sexuales: semestres superiores (OR: 2,70; $p=0,003$ ), edad mayor de 18 años (OR: $2,45 ; p=0,01)$ y el consumo de alcohol (OR: 1,83 ; $\mathrm{p}=0,03)$ (tabla 4).

\begin{tabular}{|c|c|c|c|c|c|c|c|}
\hline & \multicolumn{3}{|c|}{ Relaciones sexuales } & \multirow{2}{*}{$\begin{array}{c}\text { Bivariado } \\
\text { OR凶 }\end{array}$} & \multicolumn{3}{|c|}{ Multivariado } \\
\hline & SI & NO & Total & & IC $95 \%$ & OR⿴囗 & IC $95 \%$ \\
\hline Edad mayor de 18 & 129 & 76 & 205 & 1,20 & $0,60-2,38$ & & \\
\hline Género Masculino & 85 & 35 & 120 & $2,07^{a}$ & $1,22-3,50$ & $2,16^{c}$ & $1,22-3,83$ \\
\hline Anticonceptivos & 92 & 26 & 118 & $3,82^{b}$ & $2,19-6,68$ & $3^{\prime} 76^{b}$ & $2,12-6,66$ \\
\hline Estrato Medio/Alto & 99 & 53 & 152 & 1,38 & $0,81-2,34$ & $1,78^{d}$ & $1.0-3,19$ \\
\hline Semestre VI a X & 93 & 50 & 143 & 1,33 & $0,79-2,24$ & & \\
\hline Consumo de Alcohol & 114 & 60 & 174 & 1,60 & $0,91-2,81$ & & \\
\hline Consumo de Drogas & 7 & 0 & 7 & & & & \\
\hline
\end{tabular}

Tabla 3. Factores asociados con haber tenido relaciones sexuales no planeadas en estudiantes de Odontología. ${ }^{\mathrm{P}} \mathrm{P}=0,007,{ }^{\mathrm{b}} \mathrm{P}=0,000$, ${ }^{\mathrm{C} P}=0,008,{ }^{\mathrm{d}} \mathrm{P}=0,05$,

\begin{tabular}{lcccc}
\hline & \multicolumn{2}{c}{ Bivariado } & \multicolumn{2}{c}{ Multivariado } \\
\hline & OR凶 & IC 95\% & OR凶区 & IC 95\% \\
\hline Masculino & & & & \\
Consumo de Alcohol & $2,21^{\mathrm{a}}$ & $1,30-3,75$ & $1,85^{\mathrm{b}}$ & $2,32-13,3$ \\
Femenino & & & & \\
Edad mayor de 18 & $4,87^{\mathrm{b}}$ & $2,65-8,96$ & $2,45^{\mathrm{c}}$ & $2,18-5,08$ \\
Semestre VI a X & $4,37^{\mathrm{b}}$ & $2,51-7,59$ & $2,70^{\mathrm{a}}$ & $1,39-5,21$ \\
Consumo de Alcohol & $2,21^{\mathrm{a}}$ & $1,30-3,75$ & $1,83^{\mathrm{d}}$ & $1,03-3,25$ \\
\hline
\end{tabular}

Tabla 4. Factores asociados con haber tenido relaciones sexuales estratificado por Género en estudiantes de Odontología. ${ }^{\mathrm{a}} \mathrm{P}=0,003,{ }^{\mathrm{b}} \mathrm{P}=0,000,{ }^{\mathrm{c}} \mathrm{P}=0,01,{ }^{\mathrm{d}} \mathrm{P}=0,03$. 


\section{DISCUSIÓN}

La prevalencia de haber tenido relaciones sexuales en el presente estudio fue del $64,6 \%$, la cual se considera alta al compararse con varios estudios sobre relaciones sexuales realizados en el país en los últimos cinco años ${ }^{11-13}$. Aunque la población objeto del presente estudio tuvo un promedio de edad de 20,4 años, lo que no permite una comparación adecuada con estudios realizados en adolescentes (edades entre 12 y 17 años), es posible comparar los resultados con un estudio realizado por Ceba$\| \operatorname{los}^{7}$ (2006) en la ciudad de Santa Martha, cuya prevalencia fue del $45,6 \%$, en una población de estudiantes de medicina con edad promedio de 18,6 años. A pesar de que hoy en día es claro que a mayor edad hay más probabilidad de haber tenido relaciones sexuales ${ }^{14}$, existe una diferencia importante en estos dos resultados lo que requiere indagar sobre la influencia de los diferentes contextos involucrados.

En cuanto a la diferencia de género encontrada en el presente estudio (prevalencia de haber tenido relaciones sexuales más alta en varones que en mujeres y edad más temprana para la primera relación sexual en varones que en mujeres), los resultados son similares a algunos estudios reportados a nivel nacional en cualquier edad ${ }^{15-17}$, lo cual se podría explicar por los elementos culturales que influyen desde la crianza de varones y mujeres en este contexto. En general los varones inician a menor edad las relaciones sexuales por curiosidad, por placer o por el rol social que el hombre ha asumido siempre a nivel del mundo occidental ${ }^{18}$, lo cual hace que se acentúen más las diferencias existentes en las percepciones que tienen los dos géneros con respecto a este tópico. Por otro lado, con respecto al uso de anticonceptivos, en el presente estudio el condón fue el de mayor uso con el 30,0 \%, encontrándose diferencias en varones y mujeres. Estos resultados fueron similares a los obtenidos por Ceballos ${ }^{19}$ en 2005 en Santa Martha. Así mismo se han reportado estudios a nivel internacional con prevalencia mucho más alta. Jhonson ${ }^{20}$ en estudiantes afroamericanos, con un promedio de edad de 20 años, encontró una prevalencia de haber tenido relaciones sexuales del 90,3\% y de éstos solo el 40,0 \% reportó el uso del condón. A nivel latinoamericano, en Chile, Martínez ${ }^{21}$ reportó en estudiantes de medicina una prevalencia de haber tenido relaciones sexuales del $61,0 \%$ y de éstos el 44,0 \% manifestó haber usado protección, lo que es muy similar a los resultados del presente estudio, demostrando que todavía esta medida preventiva de protección esta lejos de ser utilizada en forma masiva por nuestra población y permitiendo inferir que siguen influyendo los aspectos culturales dentro del éxito de los programas educativos desde las escuelas.

En cuanto a las asociaciones encontradas a través de la regresión logística, es importante buscar ex-

69 plicaciones a las diferencias encontradas en cuanto al género, ya que, al hacer el análisis en forma independiente, en varones sólo el consumo de alcohol fue significativo, mientras que en mujeres, además del consumo de alcohol, se encontró fuerza estadística con la edad mayor de 18 años y estar cursando semestres superiores. Los resultados en cuanto al consumo de alcohol concuerdan con estudios realizados por Campo ${ }^{22}$ en Santa Marta, quien al analizar el género en forma independiente encontró asociación en mujeres con las variables anteriormente descritas y otras como tener información acerca del VIH y el consumo de marihuana, y en varones además de éstas con la orientación heterosexual. En el presente estudio el consumo de sustancias ilegales como marihuana y cocaína no fue estadísticamente significativo en ninguno de los dos grupos, probablemente por la baja frecuencia encontrada (1,8\%). Además, en hombres el hecho de ser heterosexual no fue un factor de influencia para haber tenido relaciones sexuales. A nivel mundial, en países como España, García ${ }^{23}$ reporta resultados por género en cuanto al comportamiento sexual en estudiantes de diversas facultades y encuentra que no hay ninguna relación, diferencia que puede explicarse por las conductas diferentes que se asumen en países desarrollados, en los cuales hay pocos patrones de conducta en cuanto a comportarse sexualmente como hombre o mujer.

A pesar de todos los estudios reportados hasta el momento sobre la prevalencia de haber tenido relaciones sexuales, hay muy pocos reportes en estudiantes universitarios colombianos, más específicamente en estudiantes de Odontología. Además, no todos tienen un análisis multivariable por género en forma independiente, lo que puede representar dificultades al momento de tomar decisiones de salud pública, resultando evidente con los resultados obtenidos. Desde esta perspectiva, estos resultados pueden ser útiles para la oficia de Bienestar Universitario de la Universidad de Cartagena, lo cual puede analizarse al momento de utilizar medidas educativas en la comunidad estudiantil de referencia.

A pesar de las limitantes de un estudio de corte transversal, se concluye que los comportamientos sexuales en estudiantes de Odontología de la ciudad de Cartagena difieren entre varones y mujeres, encontrándose también variaciones en los factores asociados y siendo más influyentes en las mujeres con respecto a su interacción para explicar el fenómeno. Esto permite hacer una lectura desde un escenario cultural diferente, pudiendo diseñarse programas educativos con una perspectiva de género para contribuir a disminuir la alta incidencia de embarazos no planificados y enfermedades de transmisión sexual en esta población. 


\section{AGRADECIMIENTOS}

A las directivas de la Facultad de Odontología de la Universidad de Cartagena por el apoyo administrativo que tuvieron con este proyecto.

A los estudiantes de $X$ Semestre que participaron en el proceso de recolección de la información dentro del desarrollo del trabajo.

\section{BIBLIOGRAFÍA}

1. Tarazona E. Estado del Arte sobre Comportamiento Sexual Adolescente. Revista Electrónica del Instituto de Psicología y Desarrollo. 2006; 3(7):86-91

2. Fernández L, Bustos L, González. Creencias, actitudes y conocimientos en educación sexual. Rev med Chile. 2000; 128(6):56-61.

3. Campo A. La televisión colombiana y el manejo de la orientación sexual no convencional. Duazary. 2009; 6(2):86-8.

4. Bustillo M. Nivel de conocimientos y prácticas de riesgo para enfermedades de transmisión sexual (ETS). Rev SIDA-ETS. 1997; 3(3):68-73.

5. Monterrosa A. Embarazo en adolescentes. El drama de las niñas madres. Revista Planificación, Población y Desarrollo (Profamilia). 1993; 10-21.

6. Encuesta Nacional de Demografía y Salud 2005. Ministerio de Protección Social República de Colombia. Bogotá D.C. Junio 2006

7. Ceballos G, Campo A, Lafaurie M, Arroyave J. Relaciones sexuales en estudiantes de un programa de Medicina de la ciudad de Santa Marta (Colombia). Duazary. 2006; 3(2):1104.

8. Normas científicas, técnicas y administrativa para la investigación en salud. Resolución 008430 de 1993. Bogotá: Ministerio de Salud República de Colombia.

9. Hosmer DW, Lemeshow S. Applied logistic regression. 2nd edition. New York: Jhon Wiley and Sons Inc; 2000.

10. Greenland S. Modeling and variable selection in epidemiologic analysis. Am J Public Health. 1989; 79:340-9.

11. Ceballos G, Campo A. Relaciones sexuales en estudiantes adolescentes de Santa Martha Colombia: Factores asociados. Duazary. 2005; 2:4-10.
12. Castillo M, Meneses M, Silva JL, Navarrete PA, Campo A. Prevalencia de relaciones sexuales en adolescentes de un colegio de Bucaramanga, Colombia. Medunab. 2003; 6:137-43.

13. Campo A, Cogollo Z, Díaz C. Comportamientos de riesgo para la salud en adolescentes estudiantes: prevalencia y factores asociados. Salud Uninorte. 2008; 24(2): 226-34.

14. Takakuma M, Nagayama T, Sakihara S, Wilcox C. Patther of Health-risk behavior among Japanese high school student. J Sch Health. 2001; 71:23-9.

15. Ceballos G, Campo A. Relaciones sexuales en adolescentes estudiantes de tres colegios privados de Santa Martha Colombia: Factores asociados. Medunab. 2005; 8:171-5.

16. Campo A, Silva JL, Meneses M, Castillo M, Navarrete PA. Factores asociados con el inicio temprano de relaciones sexuales en un colegio de Bucaramanga, Colombia. Rev Colomb Psiquiatría. 2004; 33:367-77.

17. Barrios YI, Berrio VC, Gómez EM. Relaciones sexuales en adolescentes de una institución en Cartagena, Colombia. Arch Salud. 2007; 1:39-45.

18. González F. Conocimientos, actitudes y prácticas en salud sexual y reproductiva en jóvenes entre 14 y 25 años de edad de estratos 1 y 2 del SISBEN de un municipio del departamento de Cundinamarca. Acta colombiana de Psicología. 2004; 12:59-68.

19. Ceballos G, Campo A. Prevalencia del uso del Condón en la primera relación sexual en adolescentes de Santa Martha, Colombia: diferencias por género. Medunab. 2005; 8(2):5964.

20. Jhonson R, Douglas W, Nelson A. Sexual Behaviors of African- American Male College Students and the risk of $\mathrm{VIH}$ Infection. J of the National Med Assoc. 1992; 84(10):864-8.

21. Martínez C, Bengió R, Díaz M, Arancibia M, Alves Da Costa $\mathrm{P}$, Fernández R. La sexualidad en estudiantes universitarios: investigación de comportamientos. Rev Chil Urol. 1995; 60(2):201-4.

22. Campo A, Ceballos G, Herazo E. Factores asociados por género a relaciones sexuales en adolescentes de Santa Martha, Colombia, en el año 2004. Rev Colomb Obstetr Ginecol. 2008; 59:276-84.

23. Garcia E, Fernández P, Rico R. Género y sexo como variables moduladoras del comportamiento sexual en jóvenes universitarios. Psicotherma. 2005; 17(1):49-56. 